\title{
On the Closed-loop Control of an Argon Plasma Process
}

\author{
Petar Iordanov $^{* \dagger}$, Bernard Keville ${ }^{\dagger}$, John Ringwood ${ }^{\dagger}$, Seán Doherty ${ }^{\dagger}$ and Ronan \\ Faulkner ${ }^{\ddagger}$ \\ $\dagger$ Department of Electronic Engineering \\ National University of Ireland at Maynooth \\ Maynooth, Co. Kildare, IRELAND \\ ¥ National Centre for Plasma Science and Technology \\ Dublin City University \\ Dublin 9, IRELAND
}

E-mail: *petar.iordanov@eeng.nuim.ie

\begin{abstract}
Closed-loop control of a plasma process for etching applications is discussed in this study. Plasma processes are highly nonlinear systems that typically feature complex chemical and physical reactions. A laboratory-based plasma reactor is presented in this work, and issues concerning its closed-loop control are discussed. A PID controller for the experimental plasma process is developed and its performance is analysed. The need for a more advance control methodology is studied and some appropriate control structures are proposed. Finally, a description of the practical implementation of real-time multivariable closed-loop control for the studied plasma reactor is presented.
\end{abstract}

Keywords - semiconductor manufacturing, plasma process, process control, real-time control

\section{INTRODUCTION}

Plasma processing is the most widely used chemical process in the microelectronic industry for thin film deposition and etching. In recent years, the reactive ion etching (RIE) process has received tremendous attention and nowadays it has no equal in value alternative in semiconductor processing. RIE is a low-pressure plasma system, which involves chemical and physical reactions to achieve an etching process with suitable properties such as selectivity, uniformity and anisotropy. The physical and chemical mechanisms in RIE are known to be very complex, and are currently not entirely understood. The modelling of plasma processes requires a thorough understanding of the plasma dynamics. The process is known to be sensitive to various parameters, such as chamber geometry, accuracy of measurement sensors, chemical disturbances, etc., [1].

Plasma etching is typically implemented in an open-loop manner. Open-loop control is realised by specially designed etching "recipes". The choice of an appropriate recipe is mainly determined by the requirements for selectivity and etch rate. However, due to the process sensitivity, one and the same recipe that is applied to two identical plasma etching chambers would not necessarily produce same results. From chamber to chamber, deterioration in performance may vary to a different extent and cannot be predicted a priori. The use of linear dynamical empirical models influences to a great extent the performance of the etching systems. Such models provide little process insight and are specific to an operating point of a particular reactor. Consequently, a data-based model obtained for a particular plasma chamber would not necessarily be good enough for another chamber of the same brand and specifications, even under the same operating conditions.

All of the above facts suggest that a more sophisticated control approach is needed to provide a desired level of etching performance. A more reasonable approach to model plasma processes is to exploit the information that is available about the physical and chemical interactions occurring in the process. Models based on this methodology are usually referred to as first-principles models. As opposed to data-based models, first-principles models take advantage of parameters that describe plasma chemical kinetics, chamber geometry, delivery of radio-frequency ( $\mathrm{RF}$ ) power to the plasma, actuators, sensors, etc.

The process considered in this paper is a rela- 
tively simple laboratory-based plasma reactor. A one-species argon plasma process that does not involve etching is studied. The study of a simple process like this can be considered as an initial step towards understanding the phenomena that exist in industrial plasma etching systems. The purpose of this work is to provide insight into the closedloop control of plasma variables, such as neutral density, charged particle density, charged particle flux, electron temperature, etc. In this work, attention is focused on a single plasma variable that is associated with the rate of etching in industrial plasma reactors. Control of charged particle flux, often referred to as ion flux, is illustrated in this paper. Although increase in ion flux leads to increase in etch rate, an explicit relationship between these two variables cannot always be derived.

In this study, various issues concerning the control of charged particle flux are addressed. One of the main goals is to assess the ability of conventional SISO control designs in achieving satisfactory performance for a broad operating space. It is shown that a model-based control strategy is required to tackle the highly nonlinear and nonrobust nature of this process.

The paper is organised as follows: Section 2 gives a description of an experimental inductively coupled plasma system. The use of a PID controller for this plasma process is demonstrated in Section 3. Section 4 discusses the necessity of model-based control, the selection of an appropriate control structure, and the practical implementation of such control law. Finally, conclusions and future research directions are outlined in section 5 .

\section{Description And Set-up of the BARIS Plasma Experiment}

BARIS (BAsic Radiofrequency Inductive System) chamber, [2], is an inductively coupled discharge system that generates plasma at a radio frequency (RF) of $13.56 \mathrm{MHz}$. The discharge chamber is a stainless steel cylindrical vacuum chamber of internal diameter $200 \mathrm{~mm}$ and length $900 \mathrm{~mm}$, and is illustrated in Fig. 1. The plasma chamber can be separated into two sections. The first section contains the antenna housing, the optical diagnostic windows and three $70 \mathrm{~mm}$ vacuum ports for electrical diagnostics. The second section contains a $100 \mathrm{~mm}$ pumping port for the vacuum pumps and a KF25 port for the gas inlet. BARIS consists of a helical antenna centered co-axially inside the cylindrical stainless steel discharge chamber. The antenna is isolated from the discharge inside a quartz dielectric tube. To ensure an efficient power transfer into the plasma and minimum generation of reflected waves, the plasma system is supplied with a matching network consisting of a controller and

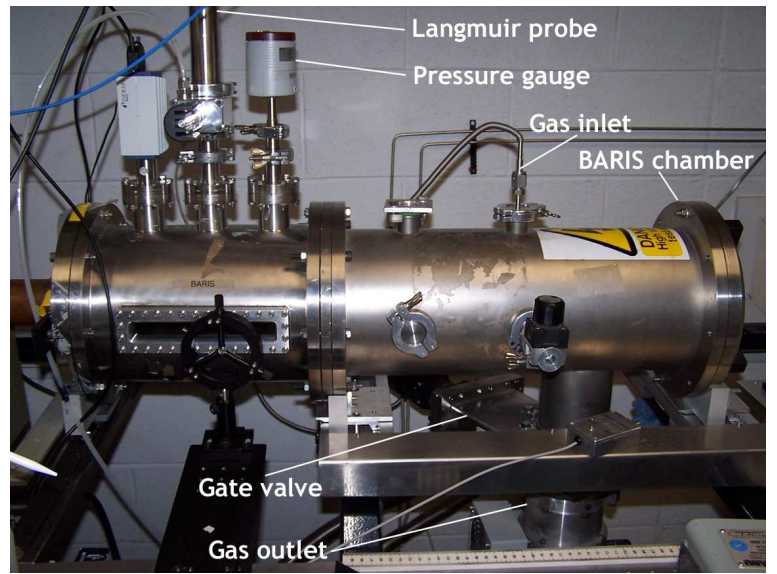

Fig. 1: BARIS Plasma Chamber

a standard "L"-configuration matching unit. The matching unit comprises of two variable capacitors manipulated by DC servomotors. The matching controller tunes the capacitors so that the source impedance is matched with the plasma discharge impedance. The plasma impedance changes as the source power, gas flow rate and pressure change, and is a complex nonlinear function of the plasma variables. Argon gas is supplied to the chamber through a mass flow controller. Gas is pumped out of the plasma chamber with the help of a turbo-molecular pump, assisted by a backing rotary vane pump. Pressure in the chamber is maintained by externally controlled gate valve mounted at the exhaust port. A number of sensors are fitted on the plasma system, including pressure gauges, power meters and a langmuir probe. The pressure gauges are capacitance manometers fitted with an interface for computer-based monitoring. The langmuir probe sensor is used for measuring the ion saturation current in the plasma. The probe tip is set to a bias voltage and the current collected on the probe tip is then measured. The actual argon ion flux is proportional to the measured ion saturation current. The RF power generator and the gate valve system are externally controlled via command sequences sent over the serial RS232 interface. The argon mass flow controller includes an analog interface that allows flow rate set points to be given from a $\mathrm{PC}$, and the actual flow rate to be read by the PC.

The BARIS plasma process is operated by an Intel Pentium D based PC including a set of analog and digital boards installed on it. The three PCI boards installed on the PC are:- Measurement and Computing analog output card PCI-DDA08/16, National Instruments analog input card PCI-6031E, and Quatech 8-port RS232 serial interface ESC-100. A block diagram of the experimental setup for the BARIS system is illustrated in Fig. 2. 


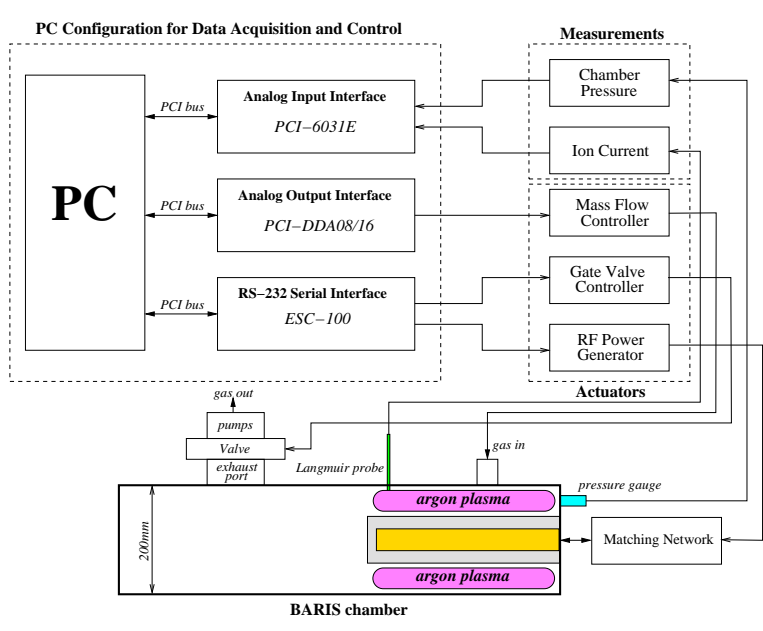

Fig. 2: Experimental setup for BARIS

A single-input single-output (SISO) control configuration is considered. The argon flow rate and the gate valve position are fixed, thus ensuring constant pressure at a specific input power. The matching controller is set to automatic mode in order to track changes in reflected power and minimise its amount in real-time. Argon ion flux is controlled by varying the level of forward power produced by the RF generator. With the above described experimental setup, a basic controller design for the BARIS process is considered in the next section.

\section{PID Controller Design}

A PID controller is now designed for the BARIS process. The control objective for this design is to provide satisfactory tracking of the ion saturation current reference trajectory. Here, "satisfactory" means that tracking performance needs to be maintained steady within the entire admissible operating space.

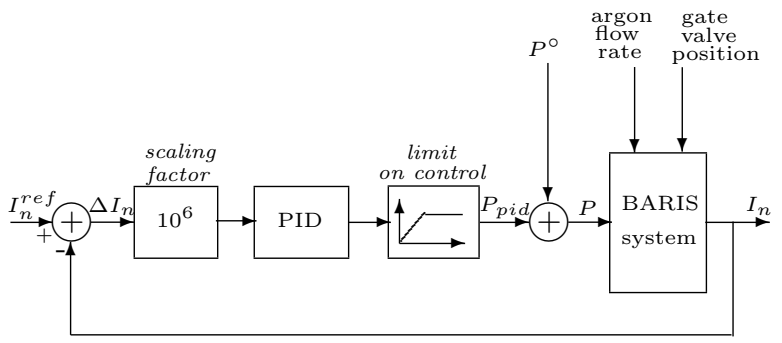

Fig. 3: Closed-loop structure with the PID controller

The controller is implemented in LabView 7.1, [3], running under Windows XP. Reliable execution of the produced VI-file was achieved at a sample time of $100 \mathrm{~ms}$. For the ion saturation current measurement, the langmuir probe was biased at $60 \mathrm{~V}$. The argon flow rate was set to $100 \mathrm{sccm}$ and the gate valve was kept fully open. Due to physical and safety restrictions, the RF power was limited to $200 \mathrm{~W}$. At this power, the ion saturation current, $I_{n}$, is about $370 \mu \mathrm{A}$. The nominal operating point is $P^{\circ}=100 \mathrm{~W}$, at which the ion saturation current is $I_{n} \approx 225 \mu \mathrm{A}$. The digital interface protocol allows only integer power levels to be set, thus the resolution of the power signal is $1 \mathrm{~W}$.

A block diagram of the closed-loop control system is shown in Fig. 3. The PID control law is described by the following continuous-time representation:

$P_{p i d}=10^{6}\left(k_{P} \Delta I_{n}+k_{I} \int \Delta I_{n} d t+k_{D} \frac{d \Delta I_{n}}{d t}\right)$

where the tracking error $\Delta I_{n}(t)$ is expressed as

$\Delta I_{n}(t)=I_{n}^{r e f}(t)-I_{n}(t)$

The RF power setpoint, $P$, is given by the following function

$P(t)=\operatorname{round}\left(P^{\circ}(t)+\min \left\{P_{p i d}(t), 100 W\right\}\right)$

Tuning of the three coefficients, $k_{P}, k_{I}$ and $k_{D}$, was done in accordance with the requirements for short response time as well as minimum overshoot and oscillations around the nominal operating point. The gain of the differential part, $k_{D}$, was found to have a very little effect on the performance, and therefore it was eliminated. For the resulting PI control law, the actual tuning was done by trial and error, and a suboptimal choice for the parameters is: $k_{P}=0.102$ and $k_{I}=0.123$. A step response around the nominal operating point is shown in Fig. 4.
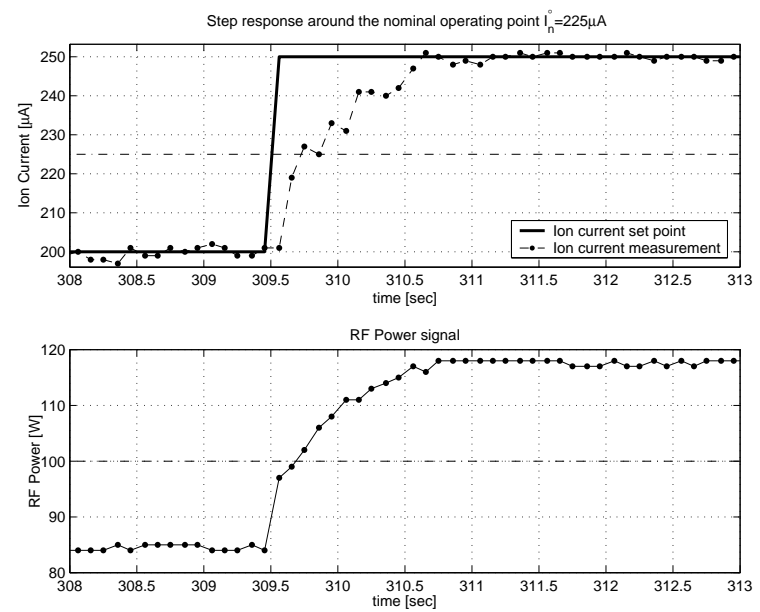

Fig. 4: Closed-loop step response around the nominal operating point

It can be seen from the figure that the response time is within 1 sec, with negligible fluctuations around the steady-state value. Although the controller works quite well around the nominal operating point, the performance deteriorates at operating points remote from the nominal operating 
point. This is illustrated in Fig. 5 where the controller is unable to provide smooth tracking from $I_{n}=300 \mu \mathrm{A}$ to $I_{n}=350 \mu \mathrm{A}$. Moving a little bit away would produce plasma instability and large oscillations around the set point.
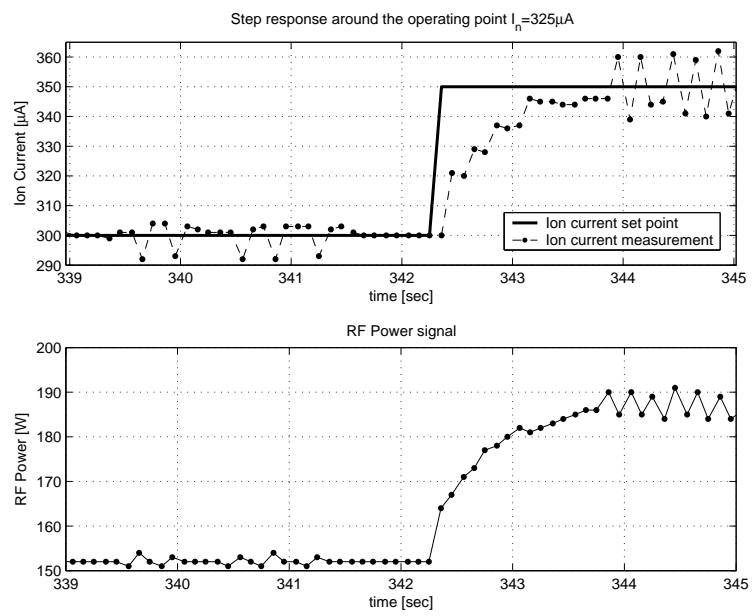

Fig. 5: Degradation in performance when moving away from the nominal operating point

It has been shown that a PID controller cannot satisfy the desired performance specifications over a large operating space. It should be noted, that the closed-loop system with the PI controller is sensitive to disturbances in flow rate and pressure, which may result in severe impedance mismatch. This simple-chemistry plasma process exhibits complex physical reactions that one needs to account for. A model-based control design must be used in order to meet the closed-loop performance specifications with respect to both set point tracking and disturbance rejection. The choice of control structure for the studied plasma process is discussed in the next section.

\section{Towards Model-BAsed Controller DESIGN AND IMPLEMENTATION}

\section{a) Modelling the Plasma Process}

A plasma model is a set of equations describing the spatioemporal evolution of the physical and chemical quantities associated with a plasma discharge. Perhaps, the simplest model that describes the interactions that occur in a plasma process is the global model. In a global model, spatial gradients within the chamber are not considered, so that the concentration of each chemical species inside the chamber is described by a single state variable. For the considered one-species plasma process, the chemical kinetics can be described by a set of four nonlinear differential equations, $[4,5,6]$ :

$$
\begin{aligned}
& \frac{d n}{d t}=k_{i} N n-\frac{A}{V} \Gamma_{n} \\
& \frac{d N}{d t}=\frac{F}{M V}-\frac{A_{E} \Gamma_{N}}{V}-k_{i} N n+\frac{\left(A-A_{E}\right) \Gamma_{n}}{V} \\
& \frac{d}{d t}\left(\frac{3}{2} n k_{B} T_{e}\right)= \frac{P}{V}-\epsilon_{i} k_{i} N n-\epsilon_{*} k_{*} N n- \\
& \frac{3}{2} \delta\left(k_{B} T_{e}-k_{B} T_{g}\right) k_{e} N n- \\
& \frac{A}{V}\left[\frac{5}{2}+\frac{1}{2} \ln \left(\frac{M}{2 \pi m}\right)\right] k_{B} T_{e} \Gamma_{n} \\
& \frac{d}{d t}\left(\frac{3}{2} N k_{B} T_{g}\right)= \frac{3}{2} \frac{F}{M V} k_{B} T_{i n}-\frac{3}{2} k_{B} T_{g} \frac{A_{E}}{V} \Gamma_{N}+ \\
& \frac{3}{2} \delta\left(k_{B} T_{e}-k_{B} T_{g}\right) k_{e} N n+ \\
& \frac{3}{2} \gamma \frac{\left(A-A_{E}\right)}{V}\left(k_{B} T_{w}-k_{B} T_{g}\right) \Gamma_{N}
\end{aligned}
$$

The four plasma variables that describe the energy and flow balance in plasma are charged particle density, $(n)$, neutral density, $(N)$, electron temperature, $\left(T_{e}\right)$, and neutral temperature, $\left(T_{g}\right)$. The charged particle flux, $\left(\Gamma_{n}\right)$, and neutral flux, $\left(\Gamma_{N}\right)$, are nonlinear functions of the plasma variables. In this description, the ionisation rate, $k_{i}$, and the excitation rate, $k_{*}$, are functions of $T_{e}$, and are given by the following expressions:

$k_{i}\left(T_{e}\right)=k_{i}^{0} e^{-\left(\frac{\epsilon_{i}}{k_{B} T_{e}}\right)}, \quad k_{*}\left(T_{e}\right)=k_{*}^{0} e^{-\left(\frac{\epsilon_{*}}{k_{B} T_{e}}\right)}$

The nonlinear dependence of the charged particle flux as function of the mass flow rate $(F)$ for a fixed deposited power $(P)$ and exhaust port size $\left(A_{E}\right)$ is illustrated in Fig. 6 .

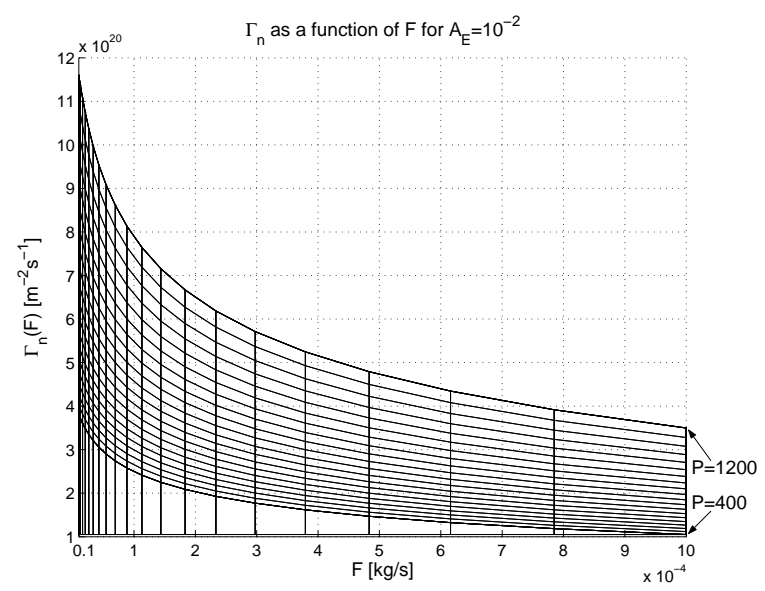

Fig. 6: Ion flux as a function of the mass flow rate

The presence of Arrhenius-type nonlinearities (such as $k_{i}\left(T_{e}\right)$ and $k_{*}\left(T_{e}\right)$ ) and complex algebraic terms involving more than two plasma variables, make the plasma model in its natural form quite difficult for analysis and control [5]. It should be noted that the global model does not explain the transfer of power from the source to the plasma. 
The representation of power deposition into the plasma is a complex function of the plasma variables and to a great extent it is determined by the antenna and chamber configuration [4]. A complete model of the plasma process also needs to incorporate models of the matching network, actuators and sensors.

\section{b) Multivariable Model-based Control Design}

A preliminary analysis of the plasma global model has already been done in a previous work, e.g. [5]. Significant variations were encountered in the global model linearised at different operating points. It has been shown that in some particular cases a Hammerstein model can be produced and a control structure for it can be devised [6]. Experimental robust control designs were generated for the global plasma model using a linear and a Hammerstein model. Although both designs demonstrated excellent performance, they worked over a relatively restricted operating space. In addition, the global model used for those designs represents significantly simplified plasma dynamics, and is virtually unrealistic. This motivates the search for a more reasonable control structure that will fully exploit the model description and ensure stable closed-loop control of the process.

A possible control solution for this process is to design a switched system based on multi-model representation of the process. The model needs to be linearised at a number of operating points and controllers providing reasonable performance in a large area around each operating point should be produced. Stability and performance sensitivity to unmodeled dynamics and parametric uncertainty could be addressed by using $\mathcal{H}_{\infty}$ or $\mu$-based robust control design, [7]. LQR optimal controllers can be generated for those operating points at which such sensitivity is not apparent.

A control design technique that has drawn much attention in the last couple of years, is the Model Predictive Control (MPC). An important advantage of MPC is that it can be used for linear as well as nonlinear models. Constraints on the manipulated and controlled variables are naturally existent in the control objective formulation. In MPC designs, the current input is determined by optimising some measure of predicted performance. MPC explicitly computes the predicted behaviour over some horizon, a feature that is lacked in most conventional control algorithms. The consideration of the reference trajectory in the MPC law generally can provide efficient command tracking. This control structure also has a native compensation for dead-times and feed-forward control is introduced in a natural way to compensate for measurable disturbances. The above features make the model predictive control a quite suitable design structure for the presented plasma process. MPC is normally associated with its real-time optimisation control law, which was specifically designated for computer-based control implementation.

\section{c) Implementation of Real-time Control}

The implementation of real-time control for the BARIS plasma system is now discussed. When building a real-time system configuration, important properties that need to be considered are reliability, computational capability and tractability to abrupt changes in the configuration. It has been shown that operating systems such as Windows fail to ensure reliable execution of deadlinecritical applications. Moreover, due to their multitasking nature, contemporary operating systems cannot efficiently provide the computational power required by applications such as LabView, [3], and MATLAB, [8]. Using a basic PC configuration, it has been found that MATLAB is not suitable for running real-time control systems due to the fact that execution deadlines at high sample rates cannot be guaranteed. Although LabView features a more reliable basis for real-time control, it is not a user-friendly platform for implementation of advanced controller designs, such as robust, optimal or model predictive control. The above comments suggest that a truly real-time control dedicated solution should be sought.

An ideal solution to implement any control structure modelled in MATLAB and/or Simulink is the MathWorks xPC Target software, [9]. xPC Target is a two-PC configuration for prototyping, testing, and deploying real-time systems using standard $\mathrm{PC}$ hardware. It is an environment that uses a target PC, separate from a host PC, for running real-time applications. A simple block diagram that illustrates the $\mathrm{xPC}$ target hardware setup for the BARIS process is shown in Fig. 7 . The host PC is used for building the executable code, while the target PC runs the deployed module. All I/O hardware that operates over actuators and sensors is installed on the target PC. Communication between both PCs is established via either RS232 interface or TCP/IP network interface (LAN, Internet or direct cross-over Ethernet connection). In this environment a desktop or laptop computer is used as a host PC with MATLAB and Simulink installed on it. After creating a Simulink model of the process, xPC target allows simulations to be performed in MATLAB in nonreal time. $\mathrm{xPC}$ Target supports numerous I/O device blocks that can be added to the model, and then with the use of Real-Time Workshop, [10], and a $\mathrm{C} / \mathrm{C}++$ compiler, an executable code is created. The executable code is downloaded from the host $\mathrm{PC}$ to the target $\mathrm{PC}$ running the $\mathrm{xPC}$ Target real-time kernel. 
An important feature of $\mathrm{xPC}$ Target is that it does not require DOS, Windows, Linux, or any another operating system on the target PC. Instead, the target $\mathrm{PC}$ can be easily booted from a floppy that includes the highly optimised xPC Target kernel. Parameters of the executable model can be tuned from the host PC while the model is still running on the target $\mathrm{PC}$. Measured and manipulated variables can be monitored and recorded on both host and target PC. xPC target kernel is a reliable platform for real-time control since its resources are fully dedicated to the code execution. Constant sample rate is maintained whenever the computational power is sufficient to do so. This software-hardware configuration is an ideal solution to implement a model-based controller for a complex nonlinear model of the studied plasma process.

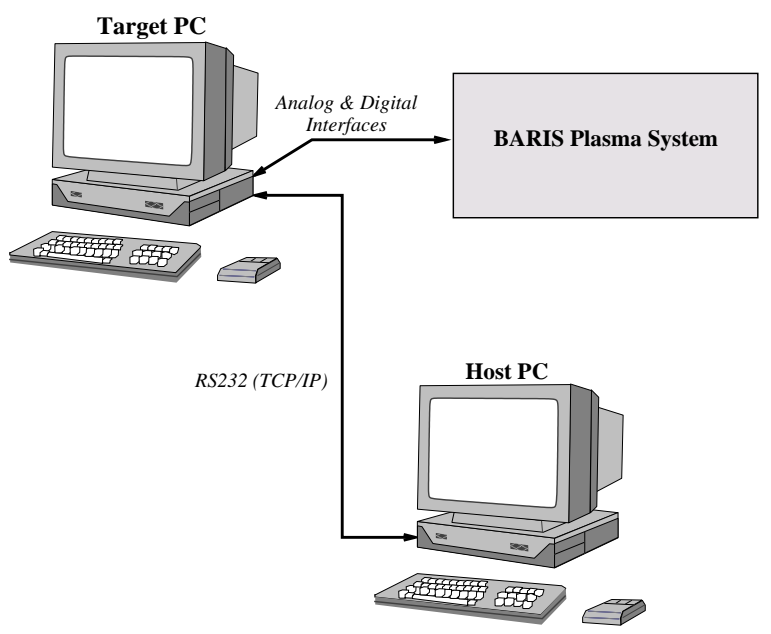

Fig. 7: xPC Target Real-time Control Configuration

\section{Conclusions And Future Work}

This paper is an example of how a basic plasma process has a rather complex dynamics that cannot be efficiently controlled by a non-model based control design. An experimental argon plasma system has been presented and a PID controller that achieves satisfactory performance around the nominal operating point has been designed. The failure of the controller to provide good level of performance away from the nominal operating point motivated the use of model-based control design. Although, at this stage, a MIMO control design has not as yet been produced, various structures that are potentially suitable for this process have been reviewed. Factors that are important with regard to the real-time closed-loop implementation are discussed and an appropriate platform for realisation has been proposed.

This study can be considered as a first step towards the ultimate objective: closed-loop control for the plasma etching process. The next step to this work is to design and implement a multivariable controller that achieves good command tracking and disturbance rejection for the control of argon ion flux. Control of ion flux and atomic oxygen density in an argon-oxygen plasma is a further step that will advance this study towards the main objective.

\section{ACKNOWLEDGEMEnts}

This work has been undertaken within the PlasMAC project, a joint collaboration between National University of Ireland at Maynooth and Dublin City University (DCU), and supported by Science Foundation Ireland (SFI). The authors would like to thank Felipe Soberon (Invent Centre, DCU). His efforts in building the experimental setup for the plasma reactor have been of immense help.

\section{REFERENCES}

[1] T.A. Badgwell, T. Breeduk, S.G. Bushman, S.W. Butler, S. Chatterjee, T.F. Edgar, A.J. Toprac and I. Trachtenberg., "Modelling and control of microelectronics material processing". Computers Chemical Engineering, 19:141, 1995.

[2] D. Doherty and P. Iordanov, "Preliminary Experimental Study of an Argon Plasma Process", Internal Report: EE/JVR/02/2005, National University of Ireland, Maynooth, 2005

[3] National Instriments, "LabVIEW Function and VI Reference Manual", 2004

[4] B. Keville, "Inductively Coupled Plasma Chamber Model for Control Analysis and Simulation", Internal Report: EE/JVR/01/2005, National University of Ireland, Maynooth

[5] P. Iordanov, J. Ringwood and S. Doherty, "Control problem classification for a plasma process", proc. of the 16th IFAC World Congress, Prague, July 2005

[6] P. Iordanov, J. Ringwood and S. Doherty, "Nonlinear Control Design for a Plasma Process", proc. of the ECC-CDC 2005 conference, pp. 2380-2385, Seville, December 2005

[7] S. Skogestad, and I. Postlethwaite, Multivariable Feedback Control : Analysis and Design, Wiley, London, 1996.

[8] The Mathworks Inc., "MATLAB: External Interfaces, User's Guide", 2002

[9] The Mathworks Inc., "xPC Target for use with real-time workshop, User's Guide", 2002

[10] The Mathworks Inc., "Real-time workshop for use with Simulink, User's Guide", 2002 